\section{Multiresistenz: \\ Wie sinnvoll sind Isolationsmaßnahmen?}

Eine Frage, die angesichts der allgemeinen Akzeptanz von Isolationsmaßnahmen bei multiresistenten Keimen irritiert. Doch Skepsis gegenüber dieser teuren und invasiven Hygienemaßnahme ist durchaus angebracht. Auf dem DAC wurden Pro und Kontra heftig diskutiert.

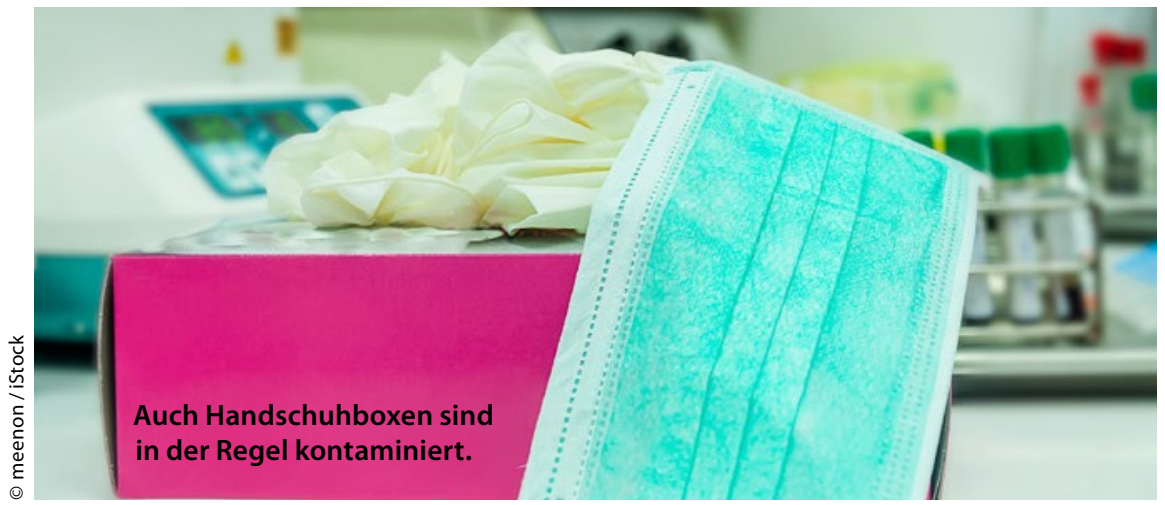

$\mathrm{D}$ r. Christian Lanckohr, Uniklinik Münster, vertrat auf dem Deutschen Anästhesiecongress (DAC) die Pro-Seite. Gleich zu Beginn spricht er die entscheidende Problematik an: „Schafft es die Isolation überhaupt, die Ausbreitung der Keime zu verhindern?" Zusätzlich gibt er zu bedenken, dass bis heute kein klarer Standard für Isolationsmaßnahmen definiert wurde.

Die Evidenz dafür, dass die Isolation die Ausbreitung der multiresistenten Erreger verhindert, ist sehr, sehr schlecht. Das zeigen zwei aktuelle Analysen aus 2015, fasst Lanckohr die Literaturlage zusammen. Auch die für die Hygienestandards verantwortliche KRINKO sieht die Sache ähnlich. Trotzdem hat sich das Gremium dazu entschieden, aufgrund einer tiefer gehenden Literaturanalyse klinische Isolationsmaßnahmen zu definieren und Empfehlungen auszusprechen. „Und diese Empfehlungen kann man jetzt nicht einfach ignorieren“, gibt er zu bedenken.

Rein hypothetisch könnte man die Isolation natürlich auch durch regelmäßige Chlorhexidinwaschungen ersetzen. Eine Strategie, die er aber aus seiner klinischen Erfahrung heraus für nicht umsetzbar hält. Außerdem kann so eine Resistenzentwicklung gegen Antiseptika induziert werden. „Zudem müssten wir kontraproduktiv. Die Oberärztin ver weist darauf, dass in Isolationseinheiten uns dann von den KRINKO-Empfehetwas schief geht?" fragt sich der teraner Intensivmediziner. Sein Fazit: Die Isolation bei Multiresistenzprobl nicht eindeutig dagegen spricht.

Prof. Dr. Maria Deja von der Charité in Berlin kritisiert die Isolationsmaßnahmen vehement. Sie seien zum Teil die Bakteriämieraten häufig ansteigen. Ursache: Mit den Einmalhandschuhen werden auch intravenöse Injektionen gegeben. „Dabei wird nicht bedacht, dass die Handschuhboxen in der Regel kontaminiert sind“, erklärt Deja. Isolationsmaßnahmen führen ihrer Meinung nach zur massiven Schädigung der Betroffenen: „Wir wissen heute, dass bei diesen Patienten weniger Temperatur gemessen wird, weniger Blutdruck gemessen wird und sie haben auch mehr Druckgeschwüre. Isolierte Patienten fallen achtmal häufiger aus dem Bett und haben Frakturen, die dann behandelt werden müssen“, so Deja.

Nach ihrer Meinung gibt es sehr wohl Evidenz dafür, dass Isolationsmaßnahmen wirkungslos sind: „Sie machen keinen Unterschied, wenn man sich die neu aufgetretenen Fälle von MRSA oder VRE anschaut. Dass da die Isolation wirklich wirkt, das geben die Studien einfach nicht her." Richtungsweisend ist für Deja eine aktuelle Analyse aus dem Jahr 2015. Diese zeigte, dass die Isolation nur bei Keimen mit hohem Transmissionsrisiko (z.B. Acinetobacter) effektiv ist. Ihr Fazit: Isolationsmaßnahmen sollten differenzierter, vor allem bei Erregern mit hohem Transmissionsrisiko, eingesetzt werden. Dr.med. Horst Gross

Isolierung von Patienten mit multiresistenten Keimen (Pro \& Con Sitzung). Dr. Christian Lanckohr (Uniklinik Münster) und Prof. Dr. med. Maria Deja (Charité Berlin), auf dem Deutschen Anästhesiecongress (DAC) 2016
Hier steht eine Anzeige. Springer 